\title{
$\beta$-Elemene inhibits proliferation through crosstalk between glia maturation factor $\beta$ and extracellular signal-regulated kinase 1/2 and impairs drug resistance to temozolomide in glioblastoma cells
}

\author{
TING-ZHUN ZHU ${ }^{1}$, XIAO-MING LI ${ }^{1}$, LI-HAN LUO ${ }^{2}$, YING-HUI XU ${ }^{3}$, \\ PENG CAO $^{1}$, YANG LIU ${ }^{1}$ and GUO-BIAO LIANG ${ }^{1}$ \\ ${ }^{1}$ Department of Neurosurgery, The General Hospital of Shenyang Military Region, Shenyang, Liaoning 110840; \\ ${ }^{2}$ Health Care Centre, Shenyang Entry-Exit Inspection and Quarantine Bureau, Shenyang, Liaoning 110016; \\ ${ }^{3}$ Department of Neurosurgery, The First Affiliated Hospital of Dalian Medical University, Dalian, Liaoning 116011, P.R. China
}

Received August 28, 2013; Accepted April 10, 2014

DOI: $10.3892 / \mathrm{mmr} .2014 .2273$

\begin{abstract}
. $\beta$-elemene, a plant-derived drug extracted from Curcuma wenyujin, has demonstrated marked antiproliferative effects on glioblastoma, while toxicity remains low. However, the underlying molecular mechanisms of the antitumor activity of $\beta$-elemene remain to be elucidated. Previously, it was identified that the glia maturation factor $\beta(\mathrm{GMF} \beta) /$ mitogen-activated protein kinase kinase (MAPK) 3/6/p38 pathway participates in the antiproliferative activity of $\beta$-elemene on glioblastoma. In the present study, in order to illustrate the association of GMF $\beta$ and the extracellular signal-regulated kinase $1 / 2$ (ERK1/2) pathway, U87 and U251 cells were treated with $\beta$-elemene at various doses and for different durations, and the expression of phosphorylated ERK1/2 (p-ERK1/2), ERK1/2, B-cell lymphoma 2 (Bcl-2), Bcl2-associated $\mathrm{X}$ and survivin was examined by western blot analysis. Following treatment with $\beta$-elemene and the ERK1/2 inhibitor PD98059, U87 cell viability was evaluated using a Cell Counting Kit-8 (CCK-8) assay, and the expression levels of $\mathrm{Bcl}-2$ and survivin were examined by western blot analysis. GMF $\beta$ was then downregulated by RNA interference in $\beta$-elemene-treated U87 cells, and the effect of this on the expression of ERK1/2 and p-ERK1/2 was determined by western blot analysis. Finally, the chemosensitisation of U87 cells to temozolomide (TMZ) through $\beta$-elemene was examined using the CCK- 8 assay. The results demonstrated that $\beta$-elemene inhibited the proliferation of U87 glioblastoma cells through the GMF $\beta$-dependent inactivation of the ERK1/2-Bcl-2/survivin pathway. Furthermore, inhibition of ERK1/2 by PD98059 enhanced the antitumor effect
\end{abstract}

Correspondence to: Dr Guo-Biao Liang, Department of Neurosurgery, The General Hospital of Shenyang Military Region, 83 Wenhua Road, Shenyang, Liaoning 110840, P.R. China

E-mail: liangguobiao6708@sohu.com

Key words: $\beta$-elemene, glioblastoma, glia maturation factor $\beta$, extracellular signal-regulated kinase $1 / 2$, temozolomide of $\beta$-elemene and impaired the expression levels of Bcl-2 and survivin. $\beta$-elemene also increased the sensitivity of U87 glioblastoma cells to the chemotherapeutic TMZ, which was synergistically enhanced by PD98059. In conclusion, these results suggested that GMF $\beta$-dependent inactivation of the ERK1/2-Bcl-2/survivin pathway mediated the antiproliferative effect of $\beta$-elemene on glioblastoma. Therefore, $\beta$-elemene is a promising chemosensitizer or adjuvant therapeutic for TMZ against glioblastoma brain tumors.

\section{Introduction}

Glioblastoma is the most common and aggressive subset of primary brain tumors, accounting for $52 \%$ of all primary intracranial tumors. Glioblastomas are lethal tumors, characterized by high chemotherapy resistance and diffuse infiltration into the brain tissue. Despite the numerous advances in the development of cancer therapeutics in recent years, the prognosis for patients with glioblastoma remains discouraging. An effective antitumor medicine is the extract from Curcuma wenyujin, and this essential oil contains a mixture of $\beta-, \gamma$ - and $\delta$-elemene (1). $\beta$-elemene (1-methyl-1-ethenyl-2,4-isopto-penyl-cyclohexane, molecular formula: $\mathrm{C}_{15} \mathrm{H}_{24}$; molecular weight: 204.34), the major active anticancer component in the elemene mixture, has demonstrated a strong antiproliferative effect and induces apoptosis in various types of tumor, including glioma, breast, liver, laryngeal, leukaemia and ovarian cancer (2-6). Previous studies by our group identified that $\beta$-elemene inhibited the proliferation of glioblastoma cells and induced cell apoptosis in vitro and in vivo (6-9). Furthermore, $\beta$-elemene exerted significant chemosensitisation effects in combination with cisplatin treatment of glioblastomas (8). However, the mechanisms underlying the antiproliferative effect of $\beta$-elemene in glioblastoma remain to be elucidated.

Glia maturation factor $\beta$ (GMF $\beta$, also known as GMF) is a $17 \mathrm{kDa}$ intracellular regulator of stress-associated signal transduction and is predominantly expressed in astrocytes in the brain. GMF $\beta$ is necessary for the growth and maturation of brain glial cells and neurons (10.11). GMF $\beta$ is able to inhibit the growth of rat C6 and human HG-1 glioblastoma cells through 
G0/G1 cell cycle arrest in vitro, reducing tumor volume and increasing the number of cells expressing glial fibrillary acidic protein (GFAP, a marker for normal astrocytes) in the tumor tissue of glioblastoma-bearing mice (12). Cellular behavioural responses to extracellular stimuli (e.g., growth factors and hormones) are also regulated by the mitogen-activated protein kinase (MAPK) pathway, which participates in the cascade reaction of MAPK kinase kinase (MKKK)-MAPK kinase (MKK)-MAPK (13). MAPK family members include extracellular signal-regulated kinase 1/2 (ERK1/2), p38 and stress-activated c-Jun N-terminal protein kinase (JNK). The ERK1/2 cascade is an evolutionarily conserved pathway and is critical in regulating multiple fundamental cellular processes, including proliferation, cell survival, differentiation, tumorigenesis and development. Constitutive activation of the ERK1/2 pathway is functionally important for cell proliferation and drug resistance in glioblastoma and ovarian cancer $(14,15)$.

It was previously identified that $\beta$-elemene is able to arrest U87 and C6 glioblastoma cells in G0/G1 phase of the cell cycle and inhibit cell proliferation through the activation of GMF $\beta$-MKK3/6-p38 and the downregulation of phosphorylated ERK1/2 (p-ERK1/2) $(6,8,16)$. Overexpression of GMF $\beta$ activates p38 and simultaneously inhibits the activity of ERK1/2 in C6 cells $(17,18)$. Therefore, it was hypothesized that there may be a potential association between the activation of GMF $\beta$ and inactivation of the ERK1/2 pathway in the antiproliferative action of $\beta$-elemene on glioblastoma cells.

In the present study, the regulatory action of GMF $\beta$ on the ERK1/2 pathway in the antiproliferative effect of $\beta$-elemene on glioblastoma was examined. The effect of $\beta$-elemene on the ERK1/2-B-cell lymphoma 2 (Bcl-2)/survivin pathway in association with GMF $\beta$ was examined. Furthermore, the effect of GMF $\beta$ silencing by transfecting small interfering (si)RNA into glioblastoma on the phosphorylation levels of ERK1/2 was assessed. In addition, the effect of $\beta$-elemene on the sensitivity of U87 glioblastoma cells to the chemotherapeutic temozolomide (TMZ) was tested. The results indicated that GMF $\beta$-ERK1/2-Bcl-2/survivin pathway may be a putative target for novel molecular therapetic strategies against glioblastoma in the future.

\section{Materials and methods}

Reagents, antibodies and cell culture. $\beta$-Elemene $(98 \%$ purity) was obtained from Jingang Pharmaceutical Co. (Dalian, China). TMZ was obtained from Sigma-Aldrich (St. Louis, MO, USA). Antibodies against p-ERK1/2, ERK1/2, GMF $\beta$, survivin, Bcl-2, Bcl-2-associated $\mathrm{X}$ protein (Bax) and GAPDH and PD98059 were obtained from Santa Cruz Biotechnology, Inc. (Santa Cruz, CA, USA). siRNAs for GMF $\beta$ and a negative control were obtained from Shanghai GenePharma Co., Ltd. (Pudongxinqu, Shanghai, China). A reverse transcription polymerase chain reaction (RT-PCR) kit was purchased from Takara Bio, Co., Ltd. (Dalian, China). The Lipofectamine 2000 transfection reagent was purchased from Invitrogen Life Technologies (Carlsbad, CA, USA). The Cell Counting kit-8 (CCK-8) was obtained from Dojindo Laboratories (Kumamoto, Japan). All of the other reagents were obtained from Sigma-Aldrich. The human U251 and
U87 glioblastoma cell lines were obtained from the Shanghai Cell Bank of the Chinese Academy of Sciences, maintained in Dulbecco's modified Eagle's medium (Hyclone, Logan, UT, USA) supplemented with $10 \%$ fetal calf serum (Invitrogen Life Technologies), $50 \mathrm{IU} / \mathrm{ml}$ penicillin (Invitrogen Life Technologies) and $50 \mathrm{mg} / \mathrm{ml}$ streptomycin (Invitrogen Life Technologies) and grown at $37^{\circ} \mathrm{C}$ in a humidified atmosphere with $5 \% \mathrm{CO}_{2}$.

Cell proliferation assay. Cell viability was evaluated by a CCK-8 assay. The cells in exponential growth phase were cultured in a 96-well culture plates and treated according to the study design. Then, $10 \mu \mathrm{l}$ of CCK- 8 was added to each well and the mixture was incubated for $4 \mathrm{~h}$ at $37^{\circ} \mathrm{C}$. The optical density (OD) of each well was measured at $450 \mathrm{~nm}$ using a spectrophotometric microplate reader (Bio-Tek Instruments, Inc., Winooski, VT, USA). Five replicate wells were designed for each cell sample. Cells were examined using an inverted microscope (ECLIPSE TE2000-U; Nikon, Tokyo, Japan).

Western blot assay. The cells were lysed with radioimmunoprecipitation assay buffer [50 mM Tris- $\mathrm{HCl}$ ( $\mathrm{pH} 7.4), 1.0 \%$ NP-40, $0.25 \%$ Na-deoxycholate, $1 \mathrm{mM}$ EDTA, $150 \mathrm{mM} \mathrm{NaCl}, 1 \mathrm{mM}$ aprotinin, $1 \mathrm{mg} / \mathrm{ml}$ phenylmethanesulfonyl fluoride, $1 \mu \mathrm{g} / \mathrm{ml}$ pepstatin and $1 \mu \mathrm{g} / \mathrm{ml}$ leupeptin]. The concentrations of total protein in the cellular extracts were measured using the bicinchoninic acid assay kit from Keygen Biotech. Co., Ltd. (Nanjing, China). Following separation by 12\% SDS-PAGE, the proteins were transferred to nitrocellulose filter membranes (Bio-Rad, Hercules, CA, USA). The membranes were blocked with $5 \%$ bovine serum albumin in Tris-buffered saline with Tween-20 at $4^{\circ} \mathrm{C}$ overnight and probed with various primary antibodies at $4^{\circ} \mathrm{C}$ overnight. This included primary antibodies against p-ERK1/2 (goat polyclonal IgG), ERK1/2 (rabbit polyclonal $\mathrm{IgG}$ ), GMF $\beta$ (mouse monoclonal $\mathrm{IgG}$ ), survivin (mouse monoclonal IgG), Bcl-2 (mouse monoclonal IgG), Bax (rabbit polyclonal IgG) and GAPDH (mouse monoclonal IgG) purchased from Santa Cruz Biotechnology, Inc. (Santa Crux, CA, USA). Followed by incubation with horseradish peroxidase-conjugated secondary antibodies (donkey anti-goat IgG, goat anti-mouse IgG and mouse anti-rabbit IgG; Santa Cruz Biotechnology, Inc.) at $37^{\circ} \mathrm{C}$ for $3 \mathrm{~h}$. The membranes were exposed to an enhanced chemiluminescence system (Amersham Biosciences, Uppsala, Sweden) and fluorescence was detected by exposing the membrane to X-ray film (Fujifilm Co., Ltd., Tokyo, Japan). The results were scanned with the Image Quant 5.2 software (Amersham Biosciences) and the gray bands were semi-quantitatively evaluated using Gel-Pro Analyzer 4.0 software (Media Cybernetics, Rockville, MD, USA). The gray values were normalized to GAPDH.

RNA silencing. The cells were plated at a density of $4 \times 10^{5}$ cells/well in six-well plates or $4 \times 10^{3} /$ well in 96 -well plates and cultured for $24 \mathrm{~h}$. siRNA oligonucleotides were transfected into glioblastoma cells with Lipofectamine 2000 according to the manufacturer's instructions. Post-transfection $(24 \mathrm{~h})$, the cells were treated with $\beta$-elemene for $24 \mathrm{~h}$. siRNA oligonucleotides were obtained from Shanghai GenePharma Co., Ltd. and specific sequences identical to those used in a previous study by our group (8) were as follows: Human 
A

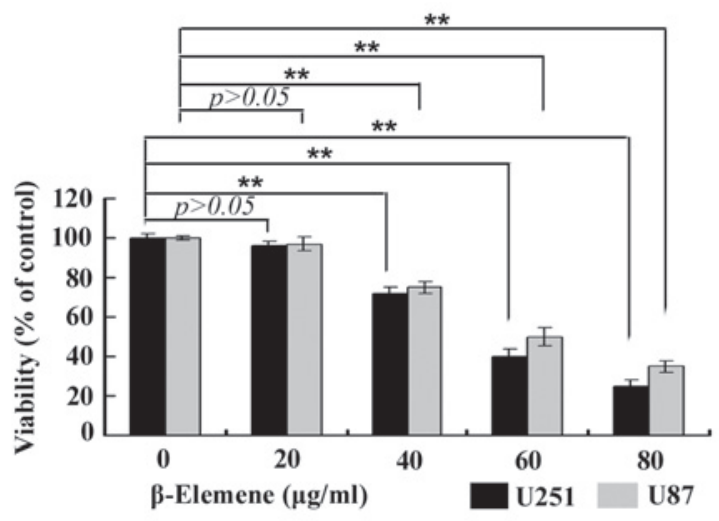

B

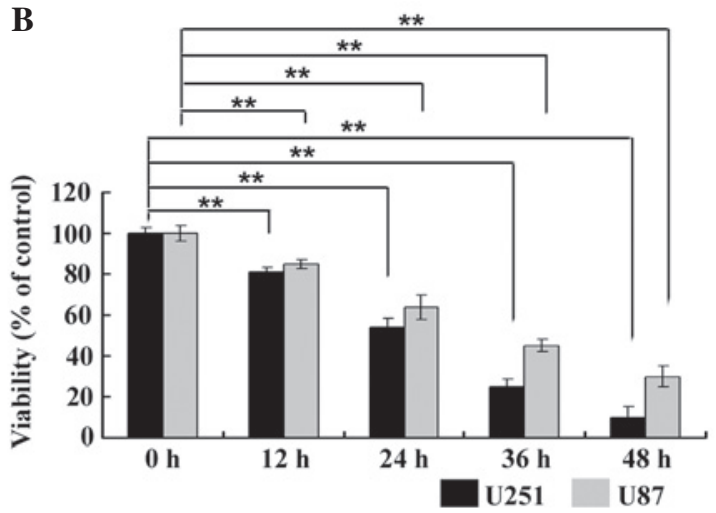

C

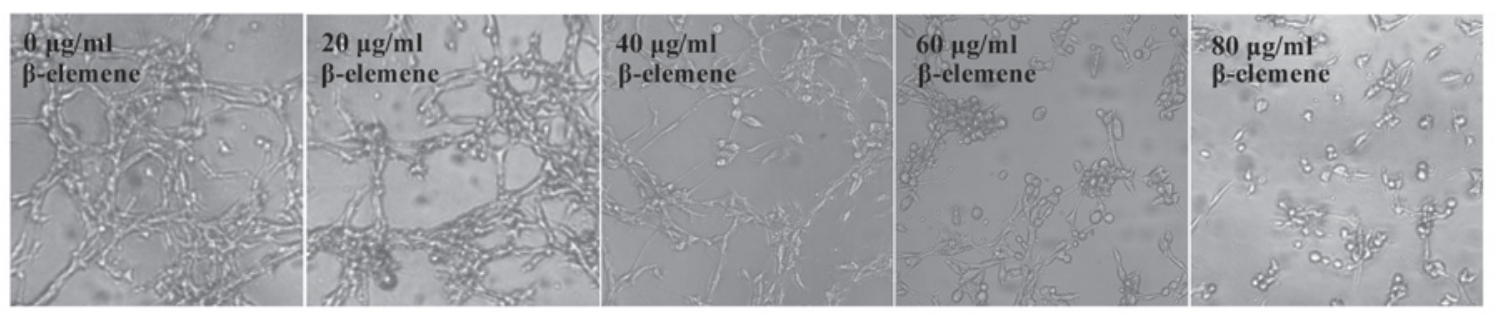

Figure 1. $\beta$-elemene inhibits the proliferation of human U87 and U251 glioblastoma cells dose- and time-dependently. (A) U87 and U251 cells were treated with $\beta$-elemene at different doses $(0,20,40,60$ and $80 \mu \mathrm{g} / \mathrm{ml})$ for $24 \mathrm{~h}$, and then, an CCK- 8 assay was performed to determine cell viability. (B) U87 and $\mathrm{U} 251$ cells were treated with $\beta$-elemene at a concentration of $60 \mu \mathrm{g} / \mathrm{ml}$ for various durations $(0,12,24,36$ and $48 \mathrm{~h})$, and cell viability was determined by a CCK- 8 assay. The viability of the cells treated with $\beta$-elemene decreased with increasing drug dose and treatment time. (C) The cells of (A) were observed by an inverted microscope (magnification, $\mathrm{x} 100)$. All of the values are represented as the mean \pm standard deviation $\left({ }^{* *} \mathrm{P}<0.01\right)$. CCK-8, Cell Counting kit- 8 .

GMF $\beta$ (NCBI reference sequence: NM_004124.2), siRNA sense 5'-GCUUCAUUGUGUAUAGUUATT-3' and antisense 5'-UAACUAUACACAAUGAAGCTT-3'. The negative control oligonucleotide sequences are as follows: Sense, 5'-UUCUCCGAACGUGUCAGGUTT-3' and antisense, 5'-ACGUGACACGUUCGGAGAATT-3'. A Basic Local Alignment Search Tool (National Center for Biotechnology Information, Bethesda, MD, USA) search was performed to ensure that the selected GMF $\beta$ siRNA sequences only targeted the GMF $\beta$ gene. The efficiency of RNA interference was determined by western blot analysis.

Statistical analysis. Values are expressed as the mean \pm standard deviation of at least three independent experiments. Statistical analysis was performed using Student's t-test. A level of $\mathrm{P}<0.05$ was considered to indicate a statistically significant difference between groups and $\mathrm{P}<0.01$ was considered to indicate a highly significant difference. Statistical analysis was performed with SPSS software version 16.0 (SPSS, Inc., Chicago, IL, USA).

\section{Results}

$\beta$-elemene inhibits the proliferation of human U87 and U251 glioblastoma cells. To evaluate the antitumor effect of $\beta$-elemene on glioblastoma cells, human U87 and U251 glioblastoma cells were treated with $\beta$-elemene at variable doses or for different durations. Cell viability was measured with a CCK-8 assay. It was identified that the viability of human U87 and U251 glioblastoma cells treated with $\beta$-elemene evidently decreased as the drug dose (Fig. 1A and C) or treatment time (Fig. 1B) increased. These results suggested that $\beta$-elemene inhibited the proliferation of human glioblastoma cells in a dose- and time-dependent manner.

$\beta$-elemene decreases the phosphorylation levels of ERK1/2 and impairs the expression of Bcl-2 and survivin. To investigate the role of the ERK1/2 signalling pathway in the antiproliferative effect of $\beta$-elemene on glioblastoma cells, the expression levels of p-ERK1/2, ERK1/2, Bcl-2, Bax and survivin were examined by western blot analysis in $\beta$-elemene-treated U87 cells (Fig. 2). $\beta$-elemene decreased the expression of p-ERK1/2, Bcl-2 and survivin in human U87 cells. By contrast, $\beta$-elemene did not affect the expression levels of total ERK1/2 and Bax.

Inhibition of ERK1/2 enhances the antiproliferative effect of $\beta$-elemene on glioblastoma in vitro. To further confirm the role of ERK1/2 inactivation in the anti-proliferative effect of $\beta$-elemene on glioblastoma cells, ERK1/2 was pretreated with $30 \mu \mathrm{M}$ PD9805 for $1 \mathrm{~h}$ in U87 cells. Following treatment with $80 \mu \mathrm{g} / \mathrm{ml} \beta$-elemene for $24 \mathrm{~h}$, U87 cell viability was measured by the CCK-8 assay (Fig. 3A) and the expression levels of Bcl-2 and survivin were detected using western blot analysis (Fig. 3B and C). The antiproliferative effect of $\beta$-elemene on glioblastoma was enhanced by PD98059, and the inhibition of ERK1/2 further decreased the expression of Bcl-2 and survivin in human U87 glioblastoma cells.

Downregulation of GMF $\beta$ decreases the inactivating activity of $\beta$-elemene on the ERK1/2 pathway. A previous study by our group reported that $\beta$-elemene inhibited the proliferation of U87 cells via activation of the GMF $\beta$ MKK3/6-p38 signalling pathway (6-8). Several studies have 
A

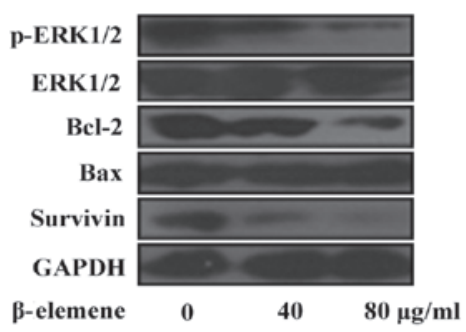

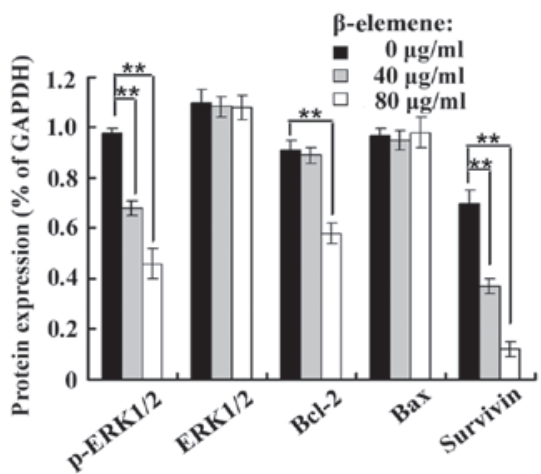

Figure 2. $\beta$-elemene decreasesthe expression of p-ERK1/2, Bcl-2 and survivin in human glioblastoma cells. U87 cells were treated with $\beta$-elemene at various concentrations $(0,40$ and $80 \mu \mathrm{g} / \mathrm{ml})$ for $24 \mathrm{~h}$, and the total protein was extracted for western blot analysis. (A) The expression levels of p-ERK1/2, ERK1/2, $\mathrm{Bcl}-2$, Bax, survivin and GAPDH were detected with specific antibodies by western blot analysis. (B) Blots were semi-quantitatively evaluated. $\beta$-elemene decreased the phosphorylation levels of ERK1/2 and impaired the expression of Bcl-2 and survivin in human U87 cells ( $\left.{ }^{* *} \mathrm{P}<0.01\right)$. However, the expression of total ERK1/2 and Bax was not affected by $\beta$-elemene. The results are representative of three independent experiments. Data are presented as the mean \pm standard deviation. p-ERK1/2, phosphorylated-extracellular signal-regulated kinase 1/2; Bcl-2, B-cell lymphoma 2; Bax, Bcl-2 associated X protein.

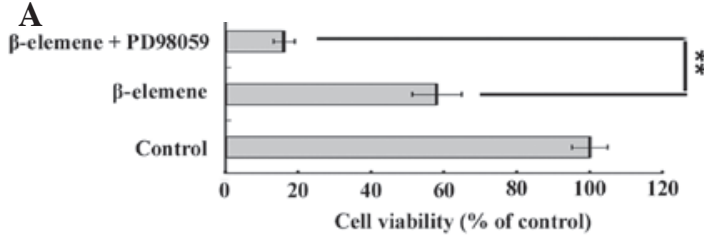

B
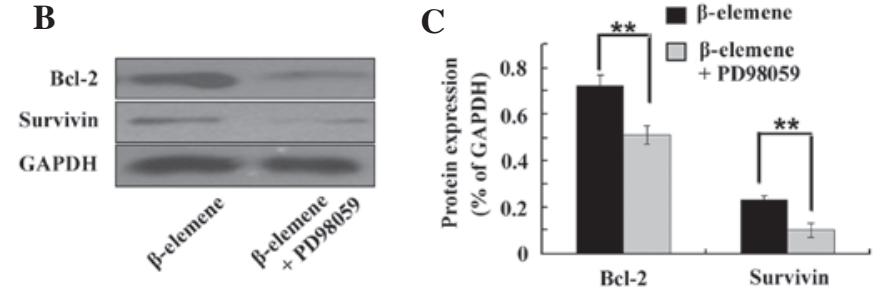

Figure 3. Inhibition of ERK1/2 by PD98059 enhances the antitumor effect of $\beta$-elemene and further decreases the expression of Bcl-2 and survivin in human U87 glioblastoma cells. U87 cells were pretreated with (or without) $30 \mu \mathrm{M}$ PD9805 for $1 \mathrm{~h}$, and then treated with $80 \mu \mathrm{g} / \mathrm{ml} \beta$-elemene for $24 \mathrm{~h}$. (A) Cell viability was measured by a CCK-8 assay (untreated U87 cells were used as the control) and (B) the expression levels of Bcl-2 and survivin were detected using western blot analysis. (C) The results of (B) were semi-quantitatively evaluated. The antiproliferative effect of $\beta$-elemene was increased by PD 98059 and the inhibition of ERK1/2 further decreased the expression of Bcl-2 and survivin. Values are presented as the mean \pm standard deviation $\left({ }^{* *} \mathrm{P}<0.01\right)$. ERK1/2, extracellular signal-regulated kinase $1 / 2$; CCK-8, Cell Counting kit-8; Bcl-2, B-cell lymphoma 2.

demonstrated that the overexpression of GMF $\beta$ simultaneously activates p38 and inhibits the activity of ERK1/2 in C6 glioblastoma cells $(17,18)$. To investigate the correlation between the GMF $\beta$ and the ERK1/2 pathway in the antiproliferative effect of $\beta$-elemene, RNA interference was performed using GMF $\beta$ siRNA to downregulate GMF $\beta$ expression in the U87 cells. The interference efficiency was determined by western blot analysis (Fig. 4A and C). U87 cells were then treated with $80 \mu \mathrm{g} / \mathrm{ml} \beta$-elemene for $24 \mathrm{~h}$ and the expression levels of p-ERK1/2 were detected by western blot analysis (Fig. 4B and D). Transfection with $53 \mathrm{nM} \mathrm{GMF} \beta$ siRNA for $24 \mathrm{~h}$ significantly decreased the expression of GMF $\beta$. The p-ERK1/2 levels were increased in the GMF $\beta$ siRNA group as compared with those in the control group. Silencing of GMF $\beta$
A

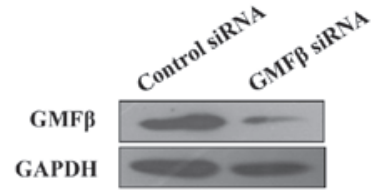

B

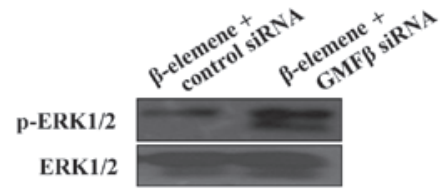

C

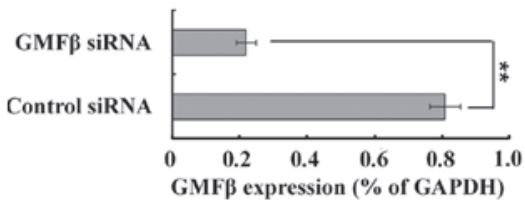

D

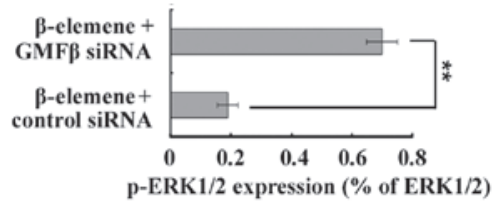

Figure 4. Silencing the expression of GMF $\beta$ impairs the inactivating action of $\beta$-elemene on the ERK1/2 pathway. (A) RNA interference was performed with $53 \mathrm{nM}$ GMF $\beta$ siRNA for $24 \mathrm{~h}$ to downregulate GMF $\beta$ expression in U87 cells. The interference efficiency was determined by western blot analysis. (B) GMF $\beta$-silenced U87 cells were treated with $80 \mu \mathrm{g} / \mathrm{ml} \beta$-elemene for $24 \mathrm{~h}$ and the expression levels of p-ERK1/2 were detected by western blot analysis. (C and D) The results of A and B, respectively, were semi-quantitatively evaluated. GMF $\beta$ siRNA significantly downregulated the expression of GMF $\beta$ and increased the p-ERK1/2 levels in U87 cells treated with $\beta$-elemene. The results are representative of three independent experiments. Values are presented as the mean \pm standard deviation $\left({ }^{* *} \mathrm{P}<0.01\right)$. GMF $\beta$, glia maturation factor $\beta ; \mathrm{p}$-ERK1/2, phosphorylated-extracellular signal-regulated kinase 1/2; siRNA, small interfering RNA.

decreased the inactivating action of $\beta$-elemene on the ERK1/2 pathway. In conclusion, the inactivation of ERK1/2 depends on GMF $\beta$ activation and mediates the antiproliferative effect of $\beta$-elemene on glioblastoma. 
$\mathbf{A}$

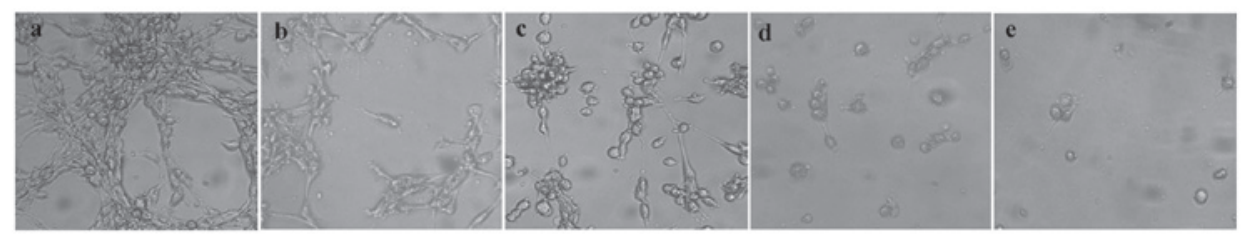

B

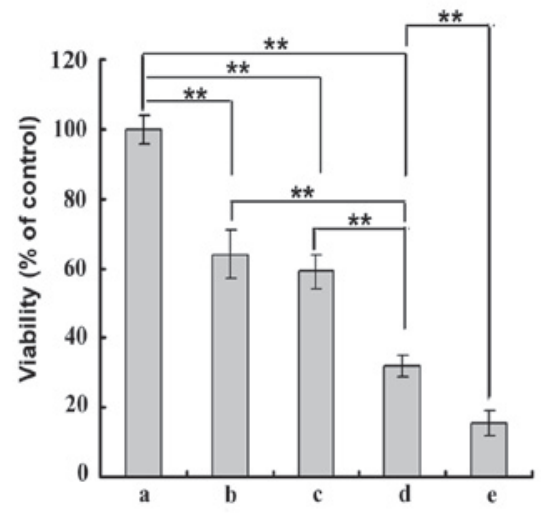

Figure 5. Treatment with $\beta$-elemene increases the sensitivity of U87 cells to TMZ-induced cytotoxicity and this chemotherapy sensitising effect is promoted by PD98059. (A) Five groups were designed: a, untreated cells; b, treated with $80 \mu \mathrm{g} / \mathrm{ml} \beta$-elemene for $24 \mathrm{~h}$; c, treated with $300 \mu \mathrm{M}$ TMZ for $24 \mathrm{~h}$; d, treated with $80 \mu \mathrm{g} / \mathrm{ml} \beta$-elemene combined with $300 \mu \mathrm{M}$ TMZ for $24 \mathrm{~h}$; and e, treated with $80 \mu \mathrm{g} / \mathrm{ml} \beta$-elemene combined with $300 \mu \mathrm{M}$ TMZ and $30 \mu \mathrm{M}$ PD98059 for $24 \mathrm{~h}$. (B) Cell viability was measured by a CCK-8 assay and quantified. The results demonstrated that the viability of the cells in group e was lower than that in groups $\mathrm{a}, \mathrm{b}, \mathrm{c}$ or $\mathrm{d}$. Treatment with $\beta$-elemene increased the sensitivity of U87 cells to TMZ-induced cytotoxicity and this chemotherapy sensitising effect was enhanced by PD98059. Values are presented as the mean \pm standard deviation $\left({ }^{* *} \mathrm{P}<0.01\right)$. TMZ, temozolomide; CCK-8, Cell Counting Kit-8.

Treatment with PD98059 promotes the chemotherapy sensitising effect of $\beta$-elemene. As previously reported, the ERK1/2 signalling pathway is associated with drug resistance in numerous types of cancer, including glioblastoma and ovarian cancer $(14,15)$. Earlier studies identified that $\beta$-elemene, as a chemosensitizer, increased the sensitivity of glioblastoma cells to cisplatin-induced cytotoxicity. To confirm whether treatment with $\beta$-elemene sensitized glioblastoma cells to TMZ-induced cytotoxicity, U87 cells were plated in 96-well plates and cultured for $24 \mathrm{~h}$. Five groups were designed: a, not treated with TMZ, $\beta$-elemene or PD98059; b, treated with $80 \mu \mathrm{g} / \mathrm{ml} \beta$-elemene for $24 \mathrm{~h}$; c, treated with $300 \mu \mathrm{M}$ TMZ for $24 \mathrm{~h}$; d, treated with $80 \mu \mathrm{g} / \mathrm{ml} \beta$-elemene combined with $300 \mu \mathrm{M}$ TMZ for $24 \mathrm{~h}$ and e, treated with $80 \mu \mathrm{g} / \mathrm{ml} \beta$-elemene combined with $300 \mu \mathrm{M}$ TMZ and $30 \mu \mathrm{M}$ PD98059 for $24 \mathrm{~h}$. U87 cells in each group were examined by inverted microscopy (Fig. 5A a-e; magnification, x100). A CCK-8 assay was conducted to determine cell viability (Fig. 5B). The viability of the cells in group $\mathrm{d}$ was lower than that in groups $\mathrm{a}, \mathrm{b}$ or $\mathrm{c}$ and the lowest viability of all of the groups was in group e. These data suggested that $\beta$-elemene increased the sensitivity of U87 cells to TMZ, and that this chemotherapy sensitising effect was enhanced by PD98059.

\section{Discussion}

Glioblastoma is the most common and lethal type of brain tumor. In spite of improvements in cancer therapeutics, the prognosis for patients with glioblastoma remains poor. The majority of chemotherapeutics are not effective in treating glioblastoma due to frequent drug resistance and the severity of the various associated side effects (19). Elemene has demonstrated potent antitumor activity against various tumor types in vitro and in vivo. $\beta$-elemene is able to arrest non-small cell lung cancer cells at the G2/M phase and induce apoptotic cell death (4). Elemene inhibited the growth of HEp-2 laryngeal cancer cells and induced cell apoptosis with decreased expression of eukaryotic translation initiation factors $4 \mathrm{E}, 4 \mathrm{G}$, basic fibroblast growth factor and vascular endothelial growth factor (20). Furthermore, $\beta$-elemene decreased the expression levels of the $\mathrm{Bcl}-2$ protein, increased cytochrome c release, and activated poly ADP-ribose polymerase and caspase-3, -7, -9 and -10 in prostate cancer cells. Concurrently, the apoptotic percentage of prostate cancer cells was increased by $\beta$-elemene in a dose- and time-dependent manner (21).

In the past ten years, numerous clinical and basic studies have been conducted on the antiproliferative effect of elemene on glioblastoma carcinoma. Elemene is able to significantly reduce the size of tumors and prolong the lifespan of patients, without serious side effects. Simultaneously, elemene has demonstrated strong antitumor activity on glioblastoma cell lines from humans, rats and glioblastoma-bearing nude mice by inducing tumor cell apoptosis, inhibiting cell proliferation and arresting cell cycle processes $(6-9,22,23)$. However, the molecular mechanisms of the antitumor activity of $\beta$-elemene are not well defined, and this hinders its application in the clinical treatment of glioblastoma.

GMF $\beta$ is an intracellular protein primarily localized in the mammalian central nervous system and is important in regulating the growth and development of glial cells and neurons. GMF $\beta$ has been identified to mediate apoptosis in glioblastoma cells and the development of an inflammatory response (24-26). Several studies have suggested that GMF $\beta$ interacts with $\mathrm{ADF} / \mathrm{cofilin}$ to promote the remodelling 
and/or disassembly of brain cortical actin structures (27). Furthermore, GMF $\beta$ inhibited the proliferation of rat $\mathrm{C} 6$ and human HG-1 glioblastoma cells and restored cell contact inhibition (12). One particular study identified that the overexpression of GMF $\beta$ in N18 neuroblastoma cells increases caspase- 3 activity and causes cytotoxicity and a loss of cell viability (28). It is widely recognized that a complex crosstalk exists between GMF $\beta$ and MAPK signalling pathways. For example, overexpression of GMF $\beta$ triggered an inflammatory response through the GMF $\beta$-p38/necrosis factor- $\kappa \mathrm{B} / \mathrm{granu}$ locyte macrophage colony-stimulating factor/tumor necrosis factor- $\alpha$, interleukin (IL)-1 $\beta$ and IL- 6 signalling pathways in astrocytes (10). Furthermore, the overexpression of GMF $\beta$ is able to activate p38 and simultaneously induce the dephosphorylation of ERK1/2 (17,18).

In recent years, numerous basic studies on the anti-glioblastoma molecular mechanism of $\beta$-elemene have been performed. It has previously been reported that $\beta$-elemene is able to arrest cells at G0/G1 in the cell cycle and activate the GMF $\beta$-MKK3/6-p38 pathway to inhibit the growth of human U87, U251 and rat C6 glioblastoma cells (6-8). In the present study, it was identified that the GMF $\beta$-dependent downregulation of $\mathrm{p}-\mathrm{ERK} 1 / 2-\mathrm{Bcl}-2 /$ survivin mediates the anti-glioblastoma proliferation effect of $\beta$-elemene. The mechanism underlying the adverse regulation of GMF $\beta$ of the ERK1/2 and p38 MAPK pathways remains elusive. GMF $\beta$ can be phosphorylated at threonine $26 /$ serine 82 by protein kinase (PK)A, at serine 71 by PKC, at threonine 26 by $\mathrm{p} 90$ ribosomal S6 kinase and at serine 52 by casein kinase II. The generation of various phosphorylation sites on GMF $\beta$ may explain its adverse modulatory actions on different MAPK members (29). According to this study, it is hypothesized that $\beta$-elemene inhibits the proliferation of human glioblastoma cells, induces cell apoptosis and causes cell cycle arrest in G0/G1 phase by activating GMF $\beta$, which activates MKK3/6-p38 and simultaneously inhibits the ERK1/2-Bcl-2/survivin pathway.

TMZ is a monofunctional alkylating agent widely used in the clinic as a first-line chemotherapeutic agent against newly diagnosed or recurrent glioblastoma $(30,31)$. However, glioblastoma cells from numerous patients often exhibit strong chemoresistance to TMZ. In the present study, it was identified that $\beta$-elemene, as a chemosensitizer, is able to increase the sensitivity of glioblastoma cells to TMZ. Furthermore, the chemosensitisation effect of $\beta$-elemene is enhanced by the ERK1/2 inhibitor PD98059, which suggests that the constitutive activation of ERK1/2 may be a drug-resistant factor in U87 glioblastoma cells. Several studies have demonstrated that ERK1/2 regulates numerous multidrug resistance proteins, including p53, O6-methylguanine-DNA-methytransferase, multidrug resistance-associated protein-1, p-glycoprotein and glutathione $S$ transferase $\pi$, to mediate chemoresistance in glioblastoma, hepatocellular, gastric, ovarian and lymphoma carcinoma types $(32-35,16)$.

In conclusion, the present study suggested that the antiproliferative effect of $\beta$-elemene on glioblastoma proceeds via the inactivation of the ERK1/2-Bcl-2/survivin pathway, which is dependent on GMF $\beta$ activation. The GMF $\beta$-ERK1/2-Bcl-2/survivin pathway may be a putative target for molecular therapy against glioblastoma. In addition, $\beta$-elemene should be investigated further, to facilitate the development of a combined therapeutic regimen with TMZ for a more efficacious strategy in the treatment of glioblastoma primary brain tumors.

\section{Acknowledgements}

This study was supported by the National Natural Science Foundation of China (no. 30740027/30471778) and China Postdoctoral Science Foundation (2012M521921). The authors are also grateful to our colleagues in our research group for their generous support.

\section{References}

1. Chen SL, You J and Wang GJ: Supercritical fluid extraction of beta-elemene under lower pressure. Se Pu 19: 179-181, 2001 (In Chinese).

2. Zhang X, Zhang Y and Li Y: $\beta$-elemene decreases cell invasion by upregulating E-cadherin expression in MCF-7 human breast cancer cells. Oncol Rep 30: 745-750, 2013.

3. Bao F, Qiu J and Zhang H: Potential role of $\beta$-elemene on histone $\mathrm{H} 1$ in the H22 ascites hepatoma cell line. Mol Med Rep 6: 185-190, 2012.

4. Wang G, Li X, Huang F, Zhao J, Ding H, Cunningham C, Coad JE, Flynn DC, Reed E and Li QQ: Antitumor effect of beta-elemene in non-small-cell lung cancer cells is mediated via induction of cell cycle arrest and apoptotic cell death. Cell Mol Life Sci 62: 881-893, 2005.

5. Li X, Wang G, Zhao J, Ding H, Cunningham C, Chen F, Flynn DC, Reed E and Li QQ: Antiproliferative effect of beta-elemene in chemoresistant ovarian carcinoma cells is mediated through arrest of the cell cycle at the G2-M phase. Cell Mol Life Sci 62: 894-904, 2005

6. Zhu T, Zhao Y, Zhang J, Li L, Zou L, Yao Y and Xu Y: $\beta$-elemene inhibits proliferation of human glioblastoma cells and causes cell-cycle G0/G1 arrest via mutually compensatory activation of MKK3 and MKK6. Int J Oncol 38: 419-426, 2011.

7. Yao YQ, Ding X, Jia YC, Huang CX, Wang YZ and Xu YH: Anti-tumor effect of beta-elemene in glioblastoma cells depends on p38 MAPK activation. Cancer Lett 264: 127-134, 2008.

8. Zhu T, Xu Y, Dong B, Zhang J, Wei Z, Xu Y and Yao Y: $\beta$-elemene inhibits proliferation of human glioblastoma cells through the activation of gliamaturation factor $\beta$ and induces sensitization to cisplatin. Oncol Rep 26: 405-413, 2011.

9. Yao YQ, Xu YH, Lu J, Zhou HY and Wang YZ: Effect of p38 MAPK on elemene-induced cell cycle arrest in C6 glioblastoma cells. Zhonghua Yi Xue Za Zhi 88: 56-58, 2008 (In Chinese).

10. Zaheer A, Zaheer S, Sahu SK, Knight S, Khosravi H, Mathur SN and Lim R: A novel role of glia maturation factor: induction of granulocyte-macrophage colony-stimulating factor and pro-inflammatory cytokines. J Neurochem 101: 364-376, 2007.

11. Thangavel R, Kempuraj D, Stolmeier D, Anantharam P, Khan M and Zaheer A: Glia maturation factor expression in entorhinal cortex of Alzheimer's disease brain. Neurochem Res 38: 1777-1784, 2013.

12. Lim R, Hicklin DJ, Ryken TC, Han XM, Liu KN, Miller JF and Baggenstoss BA: Suppression of glioma growth in vitro and in vivo by glia maturation factor. Cancer Res 46: 5241-5247, 1986.

13. Paunovic V and Harnett MM: Mitogen-activated protein kinases as therapeutic targets for rheumatoid arthritis. Drugs 73: 101-115, 2013.

14. Lo HW: Targeting Ras-RAF-ERK and its interactive pathways as a novel therapy for malignant gliomas. Curr Cancer Drug Targets 10: 840-848, 2010.

15. Liu HZ, Yu C, Yang Z, He JL, Chen WJ, Yin J, Li WM, Liu HT and Wang YX: Tubeimoside I sensitizes cisplatin in cisplatin-resistant human ovarian cancer cells (A2780/DDP) through down-regulation of ERK and up-regulation of p38 signaling pathways. Mol Med Rep 4: 985-992, 2011.

16. Zhao YS, Zhu TZ, Yao YQ, Liu RY, Wu CM, Wei ZQ, Wang W and $\mathrm{Xu}$ YH: $\beta$-elemene inhibits Hsp90/Raf-1 molecular complex inducing apoptosis of glioblastoma cells. J Neurooncol 107: 307-314, 2012 . 
17. Zaheer A and Lim R: In vitro inhibition of MAP kinase (ERK1/ERK2) activity by phosphorylated glia maturation factor (GMF). Biochemistry 35: 6283-6288, 1996.

18. Lim R and Zaheer A: In vitro enhancement of p38 mitogen-a ctivated protein kinase activity by phosphorylated glia maturation factor. J Biol Chem 271: 22953-22956, 1996.

19. Ali K, Lu Y, Das U, Sharma RK, Wiebe S, Meguro K, Sadanand V, Fourney DR, Vitali A, Kelly M, et al: Biomolecular diagnosis of human glioblastoma multiforme using Synchrotron mid-infrared spectromicroscopy. Int J Mol Med 26: 11-16, 2010.

20. Tao L, Zhou L, Zheng L and Yao M: Elemene displays anti-cancer ability on laryngeal cancer cells in vitro and in vivo. Cancer Chemother Pharmacol 58: 24-34, 2006.

21. Li QQ, Wang G, Huang F, Banda M and Reed E: Antineoplastic effect of beta-elemene on prostate cancer cells and other types of solid tumour cells. J Pharm Pharmacol 62: 1018-1127, 2010.

22. Xu YH, Dong B, Luo QZ, Zhou HY, Jia YC, Yang YF and Wang YZ: Influence of elemene on the expression of Bcl-2 family genes in rat C6 glioma cells. Zhonghua Yi Xue Za Zhi 85: 1700-1703, 2005 (In Chinese).

23. Yao YQ, Xu YH, Zhou HY, Cui CZ and Wang YZ: Role of ERK in the inhibitory effects of elemene on the proliferation of rat C6 glioblastoma cells. Tumor 27: 777-779, 2007.

24. Zaheer A, Zaheer S, Thangavel R, Wu Y, Sahu SK and Yang B: Glia maturation factor modulates beta-amyloid-induced glial activation, inflammatory cytokine/chemokine production and neuronal damage. Brain Res 1208: 192-203, 2008.

25. Zaheer A, Zaheer S, Sahu SK, Yang B and Lim R: Reduced severity of experimental autoimmune encephalomyelitis in GMF-deficient mice. Neurochem Res 32: 39-47, 2007.

26. Zaheer S, Wu Y, Bassett J, Yang B and Zaheer A: Glia maturation factor regulation of STAT expression: a novel mechanism in experimental autoimmune encephalomyelitis. Neurochem Res 32: 2123-2131, 2007.
27. Gandhi M, Smith BA, Bovellan M, Paavilainen V, Daugherty-Clarke K, Gelles J, Lappalainen P and Goode BL: GMF is a cofilin homolog that binds Arp2/3 complex to stimulate filament debranching and inhibit actin nucleation. Curr Biol 20: 861-867, 2010.

28. Zaheer A, Knight S, Zaheer A, Ahrens M, Sahu SK and Yang B: Glia maturation factor overexpression in neuroblastoma cells activates glycogen synthase kinase-3beta and caspase-3. Brain Res 1190: 206-214, 2008.

29. Zaheer A and Lim R: Protein kinase A (PKA)- and protein kinase C-phosphorylated glia maturation factor promotes the catalytic activity of PKA. J Biol Chem 272: 5183-5186, 1997.

30. Reardon DA: Treatment of elderly patients with glioblastoma. Lancet Oncol 13: 656-657, 2012.

31. Sengupta S, Marrinan J, Frishman C and Sampath P: Impact of temozolomide on immune response during malignant glioma chemotherapy. Clin Dev Immunol 2012: 831090, 2012.

32. El Azreq MA, Naci D and Aoudjit F: Collagen/ $\beta 1$ integrin signaling up-regulates the ABCC1/MRP-1 transporter in an ERK/MAPKdependent manner. Mol Biol Cell 23: 3473-3484, 2012.

33. Sato A, Sunayama J, Matsuda K, Seino S, Suzuki K, Watanabe E, Tachibana K, Tomiyama A, Kayama T and Kitanaka C: MEK-ERK signaling dictates DNA-repair gene MGMT expression and temozolomide resistance of stem-like glioblastoma cells via the MDM2-p53 axis. Stem Cells 29: 1942-1951, 2011.

34. Zhang Y, Qu XJ, Liu YP and Hou KZ: PD98059 enhancing the effects of oxaliplatin on human colorectal cancer RKO cells mediated by downregulation of GST- $\pi$ expression. Chin J Clinicians 5: 6957-6960, 2011.

35. Tomiyasu H, Watanabe M, Goto-Koshino Y, Fujino Y, Ohno K, Sugano S and Tsujimoto H: Regulation of expression of ABCB1 and LRP genes by mitogen-activated protein kinase/extracellular signal-regulated kinase pathway and its role in generation of side population cells in canine lymphoma cell lines. Leuk Lymphoma 54: 1309-1315, 2013. 